\title{
Directed VR-Representable Graphs Have Unbounded Dimension
}

\author{
Kathleen Romanik ${ }^{\star 1}$ \\ DIMACS Center for Discrete Mathematics \\ and Theoretical Computer Science \\ Rutgers, The State University of New Jersey \\ P.O. Box 1179, Piscataway, NJ 08855-1179 \\ romanik@dimacs.rutgers.edu
}

\begin{abstract}
Visibility representations of graphs map vertices to sets in Euclidean space and express edges as visibility relations between these sets. A three-dimensional visibility representation that has been studied is one in which each vertex of the graph maps to a closed rectangle in $\mathbb{R}^{3}$ and edges are expressed by vertical visibility between rectangles. The rectangles representing vertices are disjoint, contained in planes perpendicular to the $z$-axis, and have sides parallel to the $x$ or $y$ axes. Two rectangles $R_{i}$ and $R_{j}$ are considered visible provided that there exists a closed cylinder $C$ of non-zero length and radius such that the ends of $C$ are contained in $R_{i}$ and $R_{j}$, the axis of $C$ is parallel to the $z$-axis, and $C$ does not intersect any other rectangle. A graph that can be represented in this way is called VR-representable.

A VR-representation of a graph can be directed by directing all edges towards the positive $z$ direction. A directed acyclic graph $G$ has dimension $d$ if $d$ is the minimum integer such that the vertices of $G$ can be ordered by $d$ linear orderings, $<_{1}, \ldots,<_{d}$, and for vertices $u$ and $v$ there is a directed path from $u$ to $v$ if and only if $u<_{i} v$ for all $1 \leq i \leq d$. In this note we show that the dimension of the class of directed VRrepresentable graphs is unbounded.
\end{abstract}

\section{VR-Representation of Graphs}

The problem of determining a visibility representation of a graph, where the vertices of the graph map to sets in Euclidean space and the edges are expressed as visibility relations between these sets, has been widely studied (see [BETT93] for a survey). A three-dimensional visibility representation has been studied by Bose et al.[BEF $\left.{ }^{+} 94\right]$, in which each vertex of the graph maps to a closed rectangle in $\mathbf{R}^{3}$ and edges correspond to vertical visibility between rectangles.

\footnotetext{
* Supported by IRIS National Network of Centres of Excellence, NSERC, and DIMACS. DIMACS is an NSF Science and Technology Center, funded under contract STC-88-09648, and also receives support from the New Jersey Commission on Science and Technology.
} 
More formally, consider an arrangement of closed, disjoint rectangles in $\mathbf{R}^{3}$ such that the planes determined by the rectangles are perpendicular to the $z$ (vertical) axis, and the sides of the rectangles are parallel to the $x$ or $y$ axes. Two rectangles $R_{i}$ and $R_{j}$ are $\epsilon$-visible in the vertical direction if between the two rectangles there is a closed cylinder $C$ of non-zero length and radius such that the ends of $C$ are contained in $R_{i}$ and $R_{j}$, the axis of $C$ is parallel to the $z$-axis, and the intersection of $C$ with any other rectangle in the arrangement is empty. Such an arrangement is a Visibility Representation by Isothetic Rectangles in 3-Space or simply a VR-Representation of a graph $G=(V, E)$. A graph admits such a representation provided that the following hold:

- There exists a 1-1 onto correspondence between the rectangles and the vertices in $V$.

- Vertices $v_{i}$ and $v_{j}$ are adjacent in $G$ if and only if their corresponding rectangles $R_{i}$ and $R_{j}$ are $\epsilon$-visible in the vertical direction.

If a graph can be represented in this way, the graph is VR-representable.

A similar representation in two-dimensions, called an 6 -visibility representation, was defined independently by Wismath [Wis85] and Tamassia and Tollis [TT86]. In this representation vertices map to closed, disjoint, horizontal line segments in the plane, and two vertices are adjacent in the graph if and only if their corresponding segments are $\epsilon$-visible in the vertical direction. Both Wismath [Wis85] and Tamassia and Tollis [TT86] independently showed that any 2-connected planar graph has an $\epsilon$-visibility representation.

\section{Dimension of Directed Acyclic Graphs}

A directed acyclic graph $G$ has dimension $d$ if $d$ is the minimum integer such that the vertices of $G$ can be ordered by $d$ linear orderings, $<_{1}, \ldots,<_{d}$, and for vertices $u$ and $v$ there is a directed path from $u$ to $v$ if and only if $u<_{i} v$ for all $1 \leq i \leq d$ [Tro92]. A class $\mathcal{G}$ of graphs has dimension $d$ if $d$ is the largest dimension of any graph in $\mathcal{G}$.

An $\epsilon$-visibility representation of a graph can be directed by directing all edges towards the positive $y$ direction. It has been shown ([BT88, RU88]) that any graph with a directed $\epsilon$-visibility representation has dimension at most two.

A VR-representation of a graph can be directed by directing all edges towards the positive $z$ direction, yielding a directed acyclic graph. What is the maximum dimension of any graph with a directed VR-representation?

Let us denote a complete bipartite graph by $K_{m, n}$, where $m$ and $n$ are the sizes of each partition, and a complete bipartite graph with a perfect matching removed by $K_{n, n}-M$, where $n$ is the size of both partitions. Note that both partitions must have the same size for there to be a perfect matching. It is well known that the directed $K_{n, n}-M$, where all edges are directed from one partition to the other, has dimension $n$. In [BEF+94] a VR-representation was given for the directed $K_{4,4}-M$, so the class of directed VR-representable graphs has dimension at least four. 
Since a directed acyclic graph can be used to represent a partial order, work done by Rival and Urrutia [RU92] on representing ordered sets by moving convex objects in $R^{3}$ is related to our study of the dimension of VR-representable graphs.

\section{Unbounded Dimension of VR-Representable Graphs}

We now show that the dimension of the class of directed VR-representable graphs is unbounded. We show this by giving a class of graphs $\mathcal{G}=\left\{G_{n} \mid n \geq 1\right\}$ such that the dimension of $G_{n}$ is at least $n$, and then giving a directed VRrepresentation of $G_{n}$.

The directed graph $G_{n}=(V, E)$ that we construct is similar to $K_{n, n}-M$, except that some edges are replaced by directed paths. It has $4 n-2$ vertices: $V=$ $\left\{a_{1}, \ldots, a_{n}, b_{1}, \ldots, b_{n-1}, c_{1}, \ldots, c_{n-1}, d_{1}, \ldots, d_{n}\right\}$. The following is a description of the edges of $G_{n}$ :

- Each vertex $a_{i}$ is a source and has edges $\left\{\left(a_{i}, d_{j}\right) \mid j<i\right\}$ coming out of it. If $i<n$, then edge $\left(a_{i}, b_{i}\right)$ is also in the graph.

- Each vertex $b_{i}$ has one edge $\left(b_{i}, c_{i}\right)$ coming out of it.

- Each vertex $c_{i}$ has edges $\left\{\left(c_{i}, d_{j}\right) \mid j>i\right\}$ coming out of it.

- Each vertex $d_{i}$ is a sink.

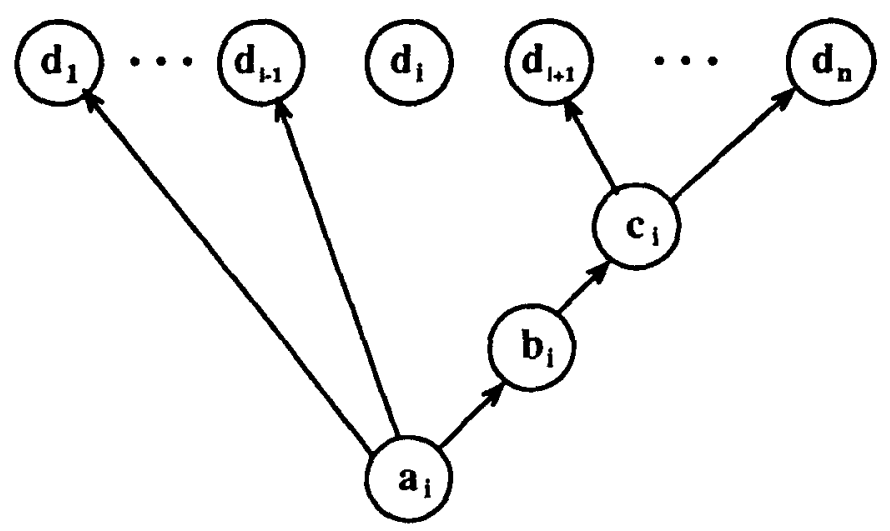

Fig. 1. Subgraph of $G_{n}$ with source vertex $a_{i}$

See Fig. 1 for an illustration of the subgraph of $G_{n}$ with source vertex $a_{i}$. In graph $G_{n}$ each vertex $a_{i}$ has an edge to each $d_{j}$, where $j<i$, and a directed path to each $d_{j}$, where $j>i$, but there is no path from $a_{i}$ to $d_{i}$. 
Lemma 1. Graph $G_{n}$ has dimension at least $n$.

Proof. We consider only the relative order of the $a_{i}$ and $d_{i}$ vertices. Since there is a directed path from $a_{i}$ to $d_{j}, j \neq i, a_{i}$ must appear before $d_{j}, j \neq i$ (i.e. $\left.a_{i}<d_{j}\right)$ in each linear ordering of the vertices. Since there is no path from $a_{i}$ to $d_{i}, d_{i}$ must appear before $a_{i}$ (i.e. $d_{i}<a_{i}$ ) in some linear ordering of the vertices. Consider a linear ordering $<_{1}$ in which $d_{i}<_{1} a_{i}$. For all other $a_{j}, j \neq i$, we must have $a_{j}<_{1} d_{i}$, and for all other $d_{j}, j \neq i$, we must have $a_{i}<_{1} d_{j}$. Thus in the ordering $<_{l}$, no other pair $a_{j}, d_{j}$ can be reversed. Since each pair $a_{j}, d_{j}$ must be reversed in some ordering, this requires at least $n$ linear orderings.

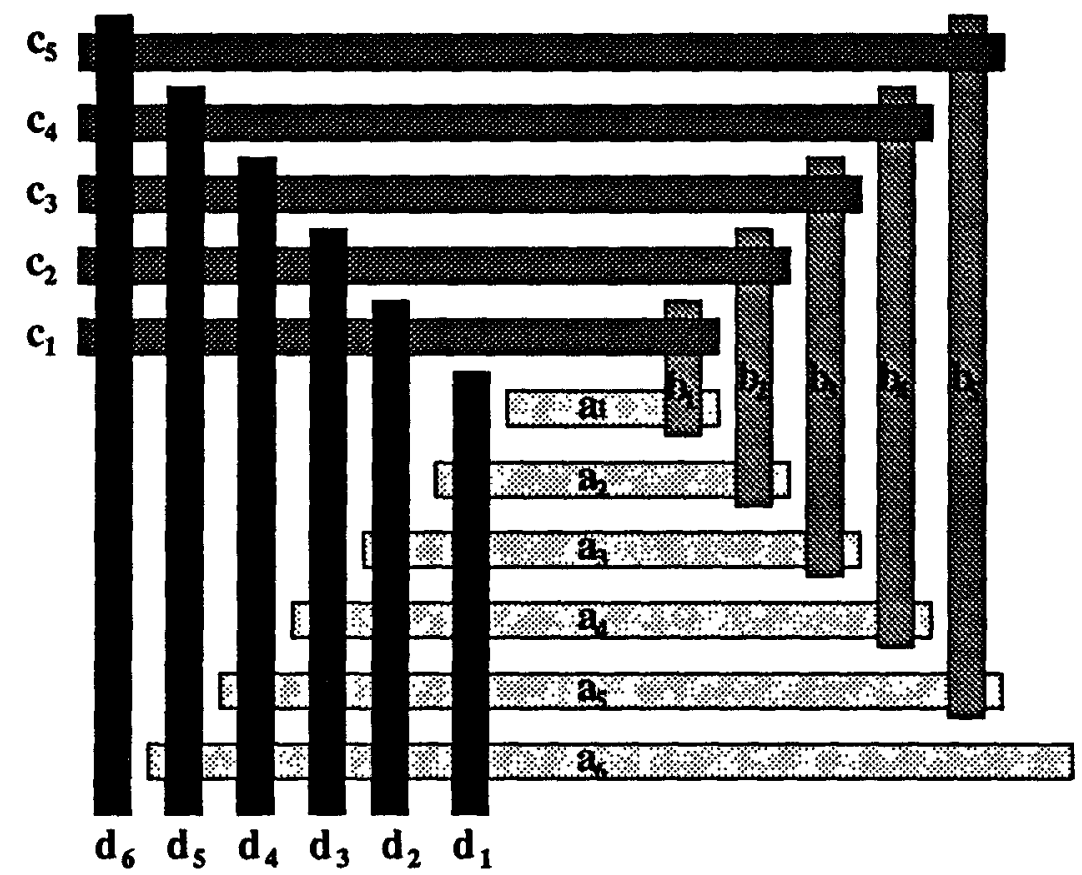

Fig. 2. VR-representation for graph $G_{n}$

We now describe a directed VR-representation for $G_{n}$. The rectangles for the $a_{i}$ vertices are contained in the plane $z=0$, those for the $b_{i}$ 's are contained in $z=1$, those for the $c_{i}$ 's are contained in $z=2$, and those for the $d_{i}$ 's are contained in $z=3$. Figure 2 illustrates the construction for $G_{6}$, where the representation is being viewed from above. It is easy to see how this construction can be extended for any $n \geq 1$. Using this VR-representation of $G_{n}$ we get the following theorem.

Theorem 2. The dimension of the class of directed VR-representable graphs is unbounded. 


\section{References}

[BEF+ 94] Prosenjit Bose, Hazel Everett, Sandor Fekete, Anna Lubiw, Henk Meijer, Kathleen Romanik, Tom Shermer, and Sue Whitesides. On a Visibility Representation for Graphs in Three Dimensions. In David Avis and Prosenjit Bose, editors, Snapshots in Computational and Discrete Geometry, Volume III. McGill University, July 1994. Technical Report SOCS-94.50.

[BETT93] Giuseppe Di Battista, Peter Eades, Roberto Tamassia, and Ioannis G. Tollis. Algorithms for Automatic Graph Drawing: An Annotated Bibliography. Technical report, Department of Computer Science, Brown University, 1993.

[BT88] Giuseppe Di Battista and Roberto Tamassia. Algorithms for Plane Representations of Acyclic Digraphs. Theoretical Computer Science, 61:175-198, 1988.

[RU88] Ivan Rival and Jorge Urrutia. Representing Orders by Translating Convex Figures in the Plane. Order 4, pages 319-339, 1988.

[RU92] Ivan Rival and Jorge Urrutia. Representing Orders by Moving Figures in Space. Discrete Mathematics, 109:255-263, 1992.

[Tro92] William T. Trotter. Combinatorics and Partially Ordered Sets: Dimension Theory. Johns Hopkins University Press, Baltimore, MD, 1992.

[TT86] Roberto Tamassia and Ioannis G. Tollis. A Unified Approach to Visibility Representations of Planar Graphs. Discrete Computational Geometry, 1:321-341, 1986.

[Wis85] Stephen K. Wismath. Characterizing Bar Line-of-Sight Graphs. In Proceedings of the First Annual Symposium on Computational Geometry, pages 147-152, Baltimore, MD, June 5-7 1985. ACM Press. 